\title{
Multiple Myeloma Vaccination Patterns in a Large Health System: A Pilot Study
}

\author{
Andinet Alemu \\ Maharaj Singh \\ Chris Blumberg \\ John O. Richards \\ Martin K. Oaks \\ Michael A. Thompson
}

Follow this and additional works at: https://aah.org/jpcrr

Part of the Oncology Commons, and the Preventive Medicine Commons

\section{Recommended Citation}

Alemu A, Singh M, Blumberg C, Richards JO, Oaks MK, Thompson MA. Multiple myeloma vaccination patterns in a large health system: a pilot study. J Patient Cent Res Rev. 2017;4:53-9. doi: 10.17294/ 2330-0698.1415

Published quarterly by Midwest-based health system Advocate Aurora Health and indexed in PubMed Central, the Journal of Patient-Centered Research and Reviews (JPCRR) is an open access, peer-reviewed medical journal focused on disseminating scholarly works devoted to improving patient-centered care practices, health outcomes, and the patient experience. 


\title{
Multiple Myeloma Vaccination Patterns in a Large Health System: A Pilot Study
}

\author{
Andinet Alemu, MD, MPH, ${ }^{1}$ Maharaj Singh, $\mathrm{PhD},{ }^{2}$ Chris Blumberg, $\mathrm{MS},{ }^{2}$ John O. Richards, $\mathrm{PhD},{ }^{2}$ \\ Martin K. Oaks, PhD, ${ }^{3}$ Michael A. Thompson, MD, PhD ${ }^{2}$ \\ ${ }^{1}$ Medical College of Wisconsin, Milwaukee, WI; ${ }^{2}$ Aurora Research Institute, Aurora Health Care, Milwaukee, Wl; \\ ${ }^{3}$ Transplant Research Laboratory, Aurora St. Luke's Medical Center, Aurora Health Care, Milwaukee, WI
}

Purpose Common reasons for hospitalization and death in patients with multiple myeloma (MM) are infections. As patients with MM are living longer and are treated with immunomodulatory drugs, there is a need to immunize against vaccine-preventable diseases and ultimately determine the efficacy of these vaccines. We evaluated vaccination practice patterns in MM patients at our health system using electronic medical records and data analytics.

Methods This institutional review board-approved study retrospectively reviewed patients with MM who visited the health system from May 2012 to May 2014. Data collected included demographics, influenza vaccination (FV) and pneumonia vaccination (PV) history, hospitalization episodes and associated costs, and duration of survival. Patients were considered PV-positive if vaccinated within 5 years prior to study. FV was defined as optimal (two FV in 2012-2014), suboptimal (one FV in 2012-2014) or none (in 2012-2014).

Results Of $411 \mathrm{MM}$ patients, $55 \%$ were male and $85 \%$ Caucasian. Nearly $58 \%$ received PV in the past 5 years. FV was $15 \%$ optimal, $52 \%$ suboptimal and $33 \%$ none. A total of 444 hospitalizations involving 204 patients were observed over 2-year follow-up. More than $\$ 23$ million was incurred from hospitalizations in the 2-year study period. There was no statistically significant difference in all-cause hospitalization and overall survival by FV and PV status.

Conclusions Despite recommendations of vaccination in multiple myeloma, our cohort had low rates of influenza and pneumonia vaccination. FV and PV status did not show any significant association with additional hospitalization or overall survival in this pilot study. Future prospective studies are needed to ascertain the immunological and clinical efficacy and effectiveness of these vaccines in immunosuppressed patients. (J Patient Cent Res Rev. 2017;4:53-59.)

Keywords multiple myeloma; vaccination; influenza; pneumococcal; hospitalization; supportive care

$\mathrm{M}$ ultiple myeloma (MM) is a cancer of plasma cells. Plasma cells are special types of terminally differentiated B-lymphocytes that are responsible for the production of antibodies to fight infection. Patients with malignancies and disorders of B-cell lineage have impaired immunity and increased risk of death from severe infection. ${ }^{1,2}$ Bacterial infections are most common, including bacteria that cause vaccine-

Correspondence: Michael A. Thompson, MD, PhD, 960 N. 12th Street, Suite 4111, Milwaukee, WI, 53233,

T: 414-219-4763, Email: Michael.A.Thompson@aurora.org preventable diseases: Streptococcus pneumoniae, Haemophilus influenzae and Neisseria meningitidis. ${ }^{3,4}$

Chemotherapy, either in the form of conventional DNA cytotoxic therapies or more putatively targeted therapies, may inhibit the immune system. The increased use of immunomodulatory drugs, such as thalidomide and lenalidomide, as well as proteasome inhibitors, such as bortezomib and carfilzomib, have led to an increase in the number of viral and fungal infections. ${ }^{5}$ Immune suppression is highest immediately after diagnosis and decreases with response to treatment. ${ }^{1}$ Augustson et al reported that $45 \%$ of the early deaths in 
MM were due to infections, primarily pneumonia and sepsis. ${ }^{6}$ A recent population-based study from Sweden by Blimark and colleagues demonstrated that patients with MM had 7-fold increased risk for a bacterial infection and 10 -fold for a viral infection. ${ }^{7}$

Vaccine-preventable diseases, like influenza and $S$. pneumoniae, are common among patients with MM. As a result, MM patients at any age are recommended to get annual inactivated influenza (FV) and pneumococcal (PV) vaccinations. ${ }^{8}$ While the safety of inactivated vaccines in patients with underlying immunosuppression has been validated in various studies, clinical outcome efficacy and effectiveness are poorly characterized, as noted in a recent review of MM vaccination studies and recommendations. ${ }^{9}$

The retrospective study presented herein assessed the pattern of FV and PV use among MM patients throughout a large health system and explored the association of vaccination status with hospitalization, cost and overall survival. It is an example of cancer care delivery research that has the potential to inform and improve existing supportive-care oncology practices.

\section{METHODS}

\section{Patient Population}

Patient data were abstracted from electronic medical records (EMR) from a large integrated health care system comprised of 15 hospitals and 20 outpatient oncology clinics. The system sees approximately 100 new analytic cases of MM per year. After institutional review board approval was obtained, records from all patients with a diagnosis of MM (as determined by ICD-9-CM code 203.0) and who had an encounter in the health system from May 15, 2012, to May 15, 2014, were reviewed using data informatics and verified by our institutional cancer registry.

Data collected included basic demographic variables, FV and PV history (as reported in the Wisconsin Immunization Registry [WIR; https://www.dhs. wisconsin.gov/immunization/wir.htm], an online database that records and tracks immunization dates of Wisconsin children and adults), hospitalization episodes, hospitalization cost, clinical outcome and censoring date. WIR was created and is operated by the state's Department of Health and Family Services "to prevent, suppress, and conduct surveillance of disease and to conduct a statewide immunization program." 10 Vaccines administered are voluntarily provided to WIR by the majority of health care providers in Wisconsin (including our health system, which submits all immunization records to WIR).

The initial data abstracted from the EMR was analyzed and published as an abstract at the American Society of Hematology (ASH) 2014 Annual Meeting. ${ }^{11}$ However, those reported data had inadvertently added non-MM patients (eg, melanoma and lymphoma) who may have been incorrectly coded. (ASH was contacted, but per ASH policy, abstracts are not retracted unless there are issues of incorrect dosages. This demonstrates one problem of relying only on ICD codes, as this information was meant for billing, not research.) For the study reported herein, the data were abstracted again and verified with our institutional cancer registry. The registry data was considered as a source of truth for patient identification. All background data, including patient demographic, diagnosis, data of diagnosis and censoring dates, were obtained from the registry. Additional data points, which included hospitalization, cost and vaccination, were obtained from EMR and WIR, respectively.

Using the EMR reporting environment, Clarity 2 (Epic Systems Corporation, Verona, WI), our institution's research analytics team performed extraction of hospitalization and cost data. The Clarity 2 database environment is a "shadow" of the Epic EMR production environment, which runs approximately 1 week behind production and is refreshed weekly. Immunization data was sourced from WIR and obtained by matching the cohort defined by the registry to the WIR database. Several identifiers were needed to match patients across the two data sources, including first name, last name, social security number, date of birth and gender. After the matching was complete, WIR provided each recorded vaccination event for the finalized patient cohort. Data was transferred between the two organizations using an encrypted flash drive sent via priority mail. The password to unlock this drive was defined by a health system administrator and provided only to a designated WIR contact.

\section{Definition of Terms}

PV (S. pneumoniae) was defined as follows: PV Yes = patient received pneumococcal vaccine within 5 years preceding the date of initial encounter with the health 
system; PV No = received no form of pneumococcal vaccine in the preceding 5 years. While various types of PV exist (eg, pneumococcal polysaccharide vaccine [PPSV23] and pneumococcal conjugate vaccine [PCV13]), ${ }^{9}$ any form of PV was considered suitable.

FV was defined as follows: None $=$ if patient didn't receive flu vaccination (in 2012-2014); Optimal $=$ if received twice over 2012-2014; and Suboptimal $=$ if received once over 2012-2014. Information on FV valency or manufacturer was not captured.

\section{Statistical Analysis}

Categorical variables were presented as percentage and counts. For all category variables, chi-squared or Fisher's test was used to compare the different groups. All continuous variables were described as mean, median and standard deviation. Kaplan-Meier method was used for survival analysis, and log-rank test was used for comparison of the groups. For multivariate timeto-event analysis, Cox proportional hazards regression was used. A two-tailed value of $\mathrm{P}<0.05$ was considered statistically significant. For all statistical analysis, SAS version 9.4 (SAS Institute, Cary, NC) was used.

\section{Outcome Variables}

Outcomes included vaccination rates and all-cause hospitalization over the 2-year study period, and overall survival. Descriptive statistics were used for cost and subgroup analyses.

\section{RESULTS}

A total of 411 patients with MM who had an encounter in our health system from May 2012 to May 2014 were included in the analysis. Median age of the study population was 68 years; other demographic characteristics are presented in Table 1.

Of $411,58 \%$ had received at least one PV (any type) in the past 5 years. Type of PV broke down as $44 \%$ PPSV23, 20\% PCV13 and 36\% unknown. There was no statistically significant difference in rate of PV when stratified by gender, age, race, hospitalization and survival time (Table 2). Mean age for patients who received PV was 67 years and for those with no $\mathrm{PV}$ was 68 years $(\mathrm{P}=0.38)$. The rate of the preferred vaccination pattern that is newly recommended, namely PCV13 followed by PPSV23, is unknown. Only $15 \%$ of all patients had optimal FV (meaning
Table 1. Patient Characteristics

\begin{tabular}{lc}
\hline Characteristic & N=411 (100\%) \\
\hline Median age & 68 years \\
Mean duration of survival & 52 months \\
Sex & \\
Male & $227(55)$ \\
Female & $184(45)$ \\
Race & \\
White & $351(85)$ \\
Black & $48(12)$ \\
Other & $12(3)$ \\
Influenza vaccination & \\
None & $135(33)$ \\
Suboptimal & $214(52)$ \\
Optimal & $62(15)$ \\
Pneumonia vaccination & \\
Yes & $237(58)$ \\
No & $174(42)$ \\
Hospitalization & \\
Yes & $207(50.4)$ \\
No & $204(49.6)$ \\
\hline
\end{tabular}

they received two consecutive seasonal FVs during the follow-up period), $52 \%$ had suboptimal $\mathrm{FV}$, and $33 \%$ did not receive any FV for three consecutive seasons. Table 3 shows stratification of FV patients by gender, age, race, hospitalization and survival time.

Approximately $50 \%$ of patients had one or more hospitalization during the 2-year period (2012-2014). Also over this period, a total of 444 hospitalizations involving 204 patients with MM were noted. Of those hospitalized, $45 \%$ had one episode, $22 \%$ had two and $32 \%$ had three or more. The total cost of hospitalization for all patients over the study duration was $\$ 23,775,512$. The average cost per hospitalization event was $\$ 116,547$, with a range of $\$ 2,337$ to $\$ 737,010$. There was no statistically significant difference in hospitalization rate when stratified by sex or race. There was no significant association between FV and PV status for all-cause hospitalization.

A total of 119 (29\%) patients died during the followup period. The survival probability of the patients with hospitalization was lower $(\mathrm{P}<0.001)$. The study was designed to be descriptive and exploratory for hospitalization and for overall survival. Median survival time was 3.4 years. A post-hoc computation with a sample of 411 patients (204 hospitalized, 207 nonhospitalized) resulted in power of more than $80 \%$ at 0.050 significance level to detect a hazard ratio of 2.29 . 
Table 2. Patient Characteristics for PV Status

\begin{tabular}{|c|c|c|c|c|}
\hline \multirow[b]{2}{*}{ Characteristic } & \multirow[b]{2}{*}{$\mathbf{N}(\%)$} & \multicolumn{2}{|c|}{ PV status } & \multirow[b]{2}{*}{$P$} \\
\hline & & Yes & No & \\
\hline Gender & & & & 0.5607 \\
\hline Male & $227(55.2)$ & $128(56.4)$ & $99(43.6)$ & \\
\hline Female & $184(44.8)$ & $109(59.2)$ & $75(40.8)$ & \\
\hline Race & & & & 0.5943 \\
\hline Black & $48(11.7)$ & $25(52.1)$ & $23(47.9)$ & \\
\hline White & $351(85.4)$ & $204(58.1)$ & $147(41.9)$ & \\
\hline Other & $12(2.9)$ & $8(66.7)$ & $4(33.3)$ & \\
\hline Hospitalization & & & & 0.7441 \\
\hline Yes & $204(49.6)$ & $116(56.9)$ & $88(43.1)$ & \\
\hline No & $207(50.4)$ & $121(58.4)$ & $86(41.6)$ & \\
\hline Mean age \pm SD & $67.3 \pm 12.1$ & $66.9 \pm 12.1$ & $67.9 \pm 12.2$ & 0.3773 \\
\hline Mean survival time* \pm SD & $52.4 \pm 50.4$ & $53.1 \pm 55.5$ & $51.5 \pm 42.7$ & 0.7546 \\
\hline
\end{tabular}

*Age was measured in years and survival time in months. PV, pneumococcal vaccination; SD, standard deviation.

Table 3. Patient Characteristics for FV Status

\begin{tabular}{|c|c|c|c|c|c|}
\hline \multirow[b]{2}{*}{ Characteristic } & \multirow[b]{2}{*}{ Total N (\%) } & \multicolumn{3}{|c|}{ FV status } & \multirow[b]{2}{*}{$P$} \\
\hline & & Optimal & Suboptimal & None & \\
\hline Gender & & & & & 0.4137 \\
\hline Male & $227(55.2)$ & $30(13.2)$ & $118(52.0)$ & 79 (34.8) & \\
\hline Female & $184(44.8)$ & $32(17.4)$ & $96(52.2)$ & $56(30.4)$ & \\
\hline Race & & & & & 0.0675 \\
\hline Black & $48(11.7)$ & $5(10.4)$ & $29(60.4)$ & $14(29.2)$ & \\
\hline White & $351(85.4)$ & $53(15.1)$ & $183(52.1)$ & $115(32.8)$ & \\
\hline Other & $12(2.9)$ & $4(33.3)$ & $2(16.7)$ & $6(50.0)$ & \\
\hline Hospitalization & & & & & 0.1561 \\
\hline Yes & $204(49.6)$ & $34(16.7)$ & $103(50.5)$ & $67(32.8)$ & \\
\hline No & $207(50.4)$ & $28(13.5)$ & $111(53.6)$ & $68(32.9)$ & \\
\hline Mean age ${ }^{\star} \pm S D$ & $67.3 \pm 12.1$ & $70.6 \pm 10.0$ & $68.1 \pm 11.5$ & $64.6 \pm 13.6$ & 0.0021 \\
\hline Mean survival time* \pm SD & $52.4 \pm 50.4$ & $57.1 \pm 63.7$ & $54.2 \pm 48.5$ & $47.4 \pm 46.2$ & 0.3564 \\
\hline
\end{tabular}

*Age was measured in years and survival time in months. FV, influenza vaccination; SD, standard deviation.

There was no statistically significant survival difference by Kaplan-Meier when the cohort was stratified separately by gender, race and vaccination status. Cox proportional hazards regression with covariates gender, race, age, hospitalization status, as well as PV and FV status, showed that hospitalized and older patients had higher risk of mortality (hazard ratio: 2.58 [95\% confidence interval: $1.74-3.83$ ] for hospitalization $[\mathrm{P}<0.001]$; hazard ratio: $1.08[95 \%$ confidence interval: $1.06-1.10]$ for age $[\mathrm{P}<0.001])$. The mean duration of survival for the cohort (calculated from date of diagnosis to last date of censoring) was 52.4 months. When stratified by race, mean duration of survival was 58.4 months for blacks, 52.6 months for whites and 23.4 months for other, but the difference was not statistically significant due in part to the very small sample of patients $(n=12)$ classified as "other" for race. 


\section{DISCUSSION}

Infection remains the most common cause of mortality among patients with MM. Respiratory infection and sepsis are quite common. The Advisory Committee on Immunization Practices (ACIP) recommendation emphasizes PV vaccine for MM patients at diagnosis. ${ }^{8}$ However, there appears to be a wide gap between the recommendation and clinical practice. We recently reviewed the history of MM vaccination studies and recommendations ${ }^{9}$ and discovered substantial variation among providers and health institutions in providing preventive vaccination in patients with cancer. This partly resulted from a lack of convincing health outcomes data and consensus in clinical efficacy of vaccination in this specific patient population.

PV has been covered by Medicare Part B since 1981. The use of the sequential PV regimen of PCV13 followed by PPSV23 has been covered since 2015. ${ }^{12}$ PV rates for non-MM adults are below public health goals. The average performance rate for the Healthcare Effectiveness Data and Information Set's PV measure is $71 \%$ in the Medicare population. In our study, only $58 \%$ of patients with MM received PV despite the recommendation to administer PV vaccination in MM patients at diagnosis. In addition, the PV given may not have aligned with the now-preferred schedule of PCV13 followed by PPSV23, per current ACIP recommendation. ${ }^{13}$

There is growing evidence that the "herd immunity" obtained against pneumococcal infection following PCV7 administration in children has contributed to decreased disease risk and antimicrobial resistance in pneumococcal invasive infection, which might have decreased the magnitude of the benefit obtained from vaccinating adult patients. ${ }^{14}$ However, it is relevant to question the effectiveness of these vaccines in patients with underlying immunosuppression like MM. This needs to be addressed in a prospective study to assess the immunologic response as well as clinical effectiveness of PV in patients with MM. ACIP has stated it will reevaluate the role of PCV13 in 2017. At that point, the timing of vaccination in adults in general, and MM patients in particular, may need to be redefined.

Patients with underlying immunosuppression are at increased risk of morbidity and mortality from influenza infection, and FV did demonstrate effectiveness in healthy individuals. ${ }^{15}$ Despite convincing data about the effectiveness of FV in healthy adults in preventing hospitalization, mortality and additional doctor visits, its effectiveness is still controversial in immunosuppressed individuals. ${ }^{15-17}$ Yet, FV is routinely recommended for most adults, particularly those with chronic respiratory, cardiac and renal disease as well as those with underlying immune suppression such as patients on active cancer and immunosuppressive medications. ${ }^{18,19}$

The mortality from influenza infection is as high as $9 \%$ in cancer patients; however, the vaccination rate in this group is lower than $50 \% .{ }^{20}$ One study by Loulergue and colleagues reported $\mathrm{FV}$ rate as low as $15 \%$ in cancer patients on chemotherapy, ${ }^{21}$ which is comparable to our findings of $15 \%$ of patients with MM who had optimal FV; however, this low rate was not significantly related to hospitalizations or overall survival. The issue of temporality/causality is a limitation to our study, as most hospitals have established policy to provide FV for patients admitted to hospital during influenza season.

A study in Uruguay showed significant reduction in hospitalization from community-acquired pneumonia (pneumococcal) and in the incidence of communityacquired pneumonia over the 3 years following universal introduction of $\mathrm{PV}$ in a pediatric population. ${ }^{22}$ However, questions about PV remain, including PCV13 and PPV23 scheduling. ${ }^{23,24}$

The effect of FV and PV has been evaluated in multiple disease states. For example, rates of $\mathrm{FV}$ in patients over 60 years old went from $<30 \%$ in 1995 to $>70 \%$ in 2005, which resulted in a decrease in all-cause mortality. ${ }^{25}$ An even greater increase in PV rate (from 0 to $>70 \%$ ) occurred over the same period of time; however, there was no discernible benefit for its use. Another study found that FV reduced exacerbations in chronic obstructive pulmonary disease, showing that the vaccine can influence patient care. ${ }^{26}$ Similar findings were observed in individuals with diabetes. Patients who had received the vaccine had reduced hospitalization rates and duration of stay. ${ }^{27}$ In a separate study, similar findings were observed that hospitalizations are reduced with the FV in patients with or without diabetes. ${ }^{28}$ 
The assessment of effectiveness of vaccines in cancer has been more complicated. In some studies there is a decrease in hospitalizations, while in others there is no difference. ${ }^{29}$ One possible explanation for these findings is that the decrease in antibody titer to the vaccine in patients with cancer $^{30}$ renders them less capable of fighting off the infection, thereby marginalizing the effectiveness of the vaccine. In multiple disease states, vaccination trends toward benefiting the patients by reducing complications, decreasing all-cause mortality and limiting the duration or number of hospital visits.

\section{Limitations}

In this study, PV status did not show any significant association with all-cause hospitalization or overall survival in patients with MM. However, there are notable potential confounders for this finding. A retrospective chart review study has several limitations related to the quality of data obtained. For this study those included: 1) retrospective nature of study and potential incompleteness of data (possible outside system admissions, etc); 2) inability to capture reason for hospitalization resulting in a potentially imperfect outcome variable of all-cause hospitalization - several studies have proved infection to be the main cause of morbidity and mortality in patients with MM, likely contributing to hospitalization and cost; ${ }^{7,14} 3$ ) lack of temporality of vaccination relative to hospitalization; 4) presence of prior or concurrent anti-MM treatments; 5) disease status (eg, stable, progressing); and 6) the sample size of 441 patients only allows for relatively large differences in health outcomes between groups to be detected and limits subset analysis.

The pilot research that generated this report established a system baseline for future prospective registry-based studies and quality improvement initiatives. In addition, gathering vaccination rates from other health systems may be informative to increase the statistical power and allow for analysis of subpopulations. Of note, we have completed a pilot sample collection study on sequential immune response metrics following vaccination in patients with MM as well as non-MM control participants.

\section{CONCLUSIONS}

Pneumococcal and influenza vaccination effectiveness is uncertain in patients with underlying immunosuppression, including those with multiple myeloma.
Various health organizations still recommend providing PV and FV in this group of patients, but the gap between the recommendations and practice may be larger for PV and FV than with other clinical preventive services. Despite low FV and PV vaccination rates in our cohort of 411 patients with MM, vaccination status was not associated with hospitalization or survival in this pilot study population. Larger studies are needed to address vaccination clinical effectiveness, surrogate markers of utility, vaccination timing, dosage and costeffectiveness.

\section{Patient-Friendly Recap}

- Multiple myeloma is a cancer of the immune system. Infections are the leading cause of death in patients diagnosed with this cancer.

- Pneumonia and influenza vaccination is recommended for these patients, but the clinical benefit is not proven.

- The authors investigated vaccination patterns in a large health system and found that many patients with multiple myeloma do not receive the recommended number of vaccinations.

- Of note, getting vaccinated did not change the patient's risk of hospitalization or death in this preliminary study.

\section{Conflicts of Interest}

Michael Thompson serves/served: as co-chair of the medical oncology multiple myeloma and indolent lymphoma committees of Via Oncology, LLC (Pittsburgh, PA); on the medical advisory boards of AIM Specialty Health (Deerfield, IL) and Doximity, Inc (San Francisco, CA); on the elotuzumab medical advisory board of Bristol-Myers Squibb Co (New York, NY); on scientific steering committees for the Connect ${ }^{\circledR}$ MDS/AML Registry of Celgene Corporation (Summit, NJ); and on the multiple myeloma registry of Millennium Pharmaceuticals, Inc (Cambridge, MA), a subsidiary of Takeda Pharmaceutical Co Ltd.

\section{Funding Sources}

Funding for this study was provided by the Vince Lombardi Cancer Foundation as an Aurora Health Care Cancer Research Award. 


\section{References}

1. Pratt G, Goodyear O, Moss P. Immunodeficiency and immunotherapy in multiple myeloma. $\mathrm{Br} J$ Haematol. 2007;138:563-79. CrossRef

2. Broder S, Humphrey R, Durm M, et al. Impaired synthesis of polyclonal (non-paraprotein) immunoglobulins by circulating lymphocytes from patients with multiple myeloma. Role of suppressor cells. N Engl J Med. 1975;293:887-92. CrossRef

3. Nucci M, Anaissie E. Infections in patients with multiple myeloma in the era of high-dose therapy and novel agents. Clin Infect Dis. 2009;49:1211-25. CrossRef

4. Teh BW, Harrison SJ, Slavin MA, Worth LJ. Epidemiology of bloodstream infections in patients with myeloma receiving current era therapy. Eur J Haematol. 2017;98:149-53. CrossRef

5. Pessoa de Magalhães RJ, Vidriales MB, Paiva B, et al. Analysis of the immune system of multiple myeloma patients achieving long-term disease control by multidimensional flow cytometry. Haematologica. 2013;98:79-86. CrossRef

6. Augustson BM, Begum G, Dunn JA, et al. Early mortality after diagnosis of multiple myeloma: analysis of patients entered onto the United Kingdom Medical Research Council trials between 1980 and 2002 - Medical Research Council Adult Leukaemia Working Party. J Clin Oncol. 2005;23:9219-26. CrossRef

7. Blimark C, Holmberg E, Mellqvist UH, et al. Multiple myeloma and infections: a population-based study on 9253 multiple myeloma patients. Haematologica. 2015;100:107-13. CrossRef

8. ACIP Adult Immunization Work Group, Bridges CB, Woods L, Coyne-Beasley T; Centers for Disease Control and Prevention. Advisory Committee on Immunization Practices (ACIP) recommended immunization schedule for adults aged 19 years and older-United States, 2013. MMWR Suppl. 2013;62:9-19.

9. Alemu A, Richards JO, Oaks MK, Thompson MA. Vaccination in multiple myeloma: review of current literature. Clin Lymphoma Myeloma Leuk. 2016;16:495-502. CrossRef

10. Wisconsin Department of Health and Family Services. Wisconsin Immunization Registry (WIR) security and confidentiality agreement. https://www.dhfswir.org/docs/Provider\%20-\%20 WIR\%20Security\%20and\%20Confidentiality\%20Agreement. pdf. Accessed December 21, 2016.

11. Alemu AW, Richards JO, Singh M, et al. Multiple myeloma vaccination patterns from a large health system: an example of cancer care delivery research (CCDR). (abstr.) Blood. 2014;124(21):5677.

12. Centers for Medicare \& Medicaid Services. Pneumococcal vaccination update from CMS (Jan. 29, 2015). https:// www.cms.gov/Medicare/Prevention/PrevntionGenInfo/ Health-Observance-Mesages-New-Items/2015-01-29Pneumococcal.html. Accessed December 21, 2016.

13. Tomczyk S, Bennett NM, Stoecker C, et al. Use of 13-valent pneumococcal conjugate vaccine and 23-valent pneumococcal polysaccharide vaccine among adults aged $\geq 65$ years: recommendations of the Advisory Committee on Immunization Practices (ACIP). MMWR Morb Mortal Wkly Rep. 2014;63:822-5.

14. Blasi F, Mantero M, Santus P, Tarsia P. Understanding the burden of pneumococcal disease in adults. Clin Microbiol Infect. 2012;18 Suppl 5:7-14. CrossRef

15. Kunisaki KM, Janoff EN. Influenza in immunosuppressed populations: a review of infection frequency, morbidity, mortality, and vaccine responses. Lancet Infect Dis. 2009;9:493-504. $\underline{\text { CrossRef }}$
16. Fry AM, Kim IK, Reed C, et al. Modeling the effect of different vaccine effectiveness estimates on the number of vaccine-prevented influenza-associated hospitalizations in older adults. Clin Infect Dis. 2014;59:406-9. CrossRef

17. Nichol KL, Nordin J, Mullooly J, Lask R, Fillbrandt K, Iwane M. Influenza vaccination and reduction in hospitalizations for cardiac disease and stroke among the elderly. $N$ Engl $J$ Med. 2003;348:1322-32. CrossRef

18. Melcher L. Recommendations for influenza and pneumococcal vaccinations in people receiving chemotherapy. Clin Oncol (R Coll Radiol). 2005;17:12-5. CrossRef

19. Löbermann, M, Boršo D, Hilgendorf I, Fritzsche C, Zettl UK, Reisinger EC. Immunization in the adult immunocompromised host. Autoimmun Rev. 2012;11:212-8. CrossRef

20. Cooksley CD, Avritscher EB, Bekele BN, Rolston KV, Geraci JM, Elting LS. Epidemiology and outcomes of serious influenza-related infections in the cancer population. Cancer. 2005;104:618-28. CrossRef

21. Loulergue P, Mir O, Alexandre J, Ropert S, Goldwasser F, Launay O. Low influenza vaccination rate among patients receiving chemotherapy for cancer. Ann Oncol. 2008;19:1658. $\underline{\text { CrossRef }}$

22. Pírez MC, Algorta G, Chamorro F, et al. Changes in hospitalizations for pneumonia after universal vaccination with pneumococcal conjugate vaccines $7 / 13$ valent and haemophilus influenzae type $\mathrm{b}$ conjugate vaccine in $\mathrm{a}$ pediatric referral hospital in Uruguay. Pediatr Infect Dis $J$. 2014;33:753-9. CrossRef

23. Bonten MJ, Huijts SM, Bolkenbaas M, et al. Polysaccharide conjugate vaccine against pneumococcal pneumonia in adults. N Engl J Med. 2015;372:1114-25. CrossRef

24. Shorr AF. PCV13 vs placebo: efficacy tested in new trial. Medscape Pulmonary Medicine (April 15, 2015). http://www.medscape.com/ viewarticle/843007. Accessed December 21, 2016.

25. Schembri S, Morant S, Winter JH, MacDonald TM. Influenza but not pneumococcal vaccination protects against all-cause mortality in patients with COPD. Thorax. 2009;64:567-72. CrossRef

26. Poole PJ, Chacko E, Wood-Baker RW, Cates CJ. Influenza vaccine for patients with chronic obstructive pulmonary disease. Cochrane Database Syst Rev. 2006;(1):CD002733. CrossRef

27. Wang CS, Wang ST, Lai CT, Lin LJ, Lee CT, Chou P. Reducing major cause-specific hospitalization rates and shortening hospital stays after influenza vaccination. Clin Infect Dis. 2004;39:1604-10. CrossRef

28. Heymann AD, Shapiro Y, Chodick G, et al. Reduced hospitalizations and death associated with influenza vaccination among patients with and without diabetes. Diabetes Care. 2004;27:2581-4. CrossRef

29. Eliakim-Raz N, Vinograd I, Zalmanovici Trestioreanu A, Leibovici L, Paul M. Influenza vaccines in immunosuppressed adults with cancer. Cochrane Database Syst Rev. 2013;(10):CD008983. CrossRef

30. Berglund A, Willén L, Grödeberg L, Skattum L, Hagberg H, Pauksens $\mathrm{K}$. The response to vaccination against influenza A(H1N1) 2009, seasonal influenza and Streptococcus pneumoniae in adult outpatients with ongoing treatment for cancer with and without rituximab. Acta Oncol. 2014;53:1212-20. $\underline{\text { CrossRef }}$

(C) 2017 Aurora Health Care, Inc. 